\title{
A QoS-aware Adaptive Coloring Scheduling Algorithm for Co-located WBANs
}

\author{
Jingxian Wang', Yongmei Sun ${ }^{1}$, Shuyun $\mathrm{Luo}^{2}$, and Yuefeng $\mathrm{Ji}^{1}$ \\ ${ }^{1}$ School of Information and Communication Engineering, Beijing University of Posts and Telecommunications \\ Beijing, 100876 - China \\ [e-mail: wangjingxian@bupt.edu.cn,ymsun@bupt.edu.cn, jyf@bupt.edu.cn] \\ ${ }^{2}$ School of Information and Technology, Zhejiang Sci-Tech University \\ Hangzhou 310018 - China \\ [e-mail: shuyunluo@zstu.edu.cn] \\ *Corresponding author: Yongmei Sun
}

Received March 30, 2017; revised March 21, 2018; accepted July 13, 2018;

published December 31, 2018

\begin{abstract}
Interference may occur when several co-located wireless body area networks (WBANs) share the same channel simultaneously, which is compressed by resource scheduling generally. In this paper, a QoS-aware Adaptive Coloring (QAC) scheduling algorithm is proposed, which contains two components: interference sets determination and time slots assignment. The highlight of QAC is to determine the interference graph based on the relay scheme and adapted to the network QoS by multi-coloring approach. However, the frequent resource assignment brings in extra energy consumption and packet loss. Thus we come up with a launch condition for the QAC scheduling algorithm, that is if the interference duration is longer than a threshold predetermined, time slots rescheduling is activated. Furthermore, based on the relative distance and moving speed between WBANs, a prediction model for interference duration is proposed. The simulation results show that compared with the state-of-the-art approaches, the QAC scheduling algorithm has better performance in terms of network capacity, average delay and resource utility.
\end{abstract}

Keywords: Wireless body area networks, interference mitigation, resource scheduling, QoS-aware adaptive coloring, relay scheme, multi-coloring 


\section{Introduction}

A Wireless Body Area Network (WBAN) is composed of a set of sensor nodes, which can be placed in, on or around human body to supervise the physiological conditions, and a coordinator, e.g. a PAD or a smartphone, to collect the information from sensor nodes and deliver it to the remote control centers, such as hospitals, by using wireless communication technologies, for instance, WiFi, 4G, etc. [1][2][3]. Evolved from Wireless Sensor Networks (WSNs), WBANs have the characteristics of frequent change of network topologies and dynamic locations due to users' movement. Thus, WBANs have high probability to meet with each other, especially for high density popularity, which has a bad impact on the network performance and results in the problem of energy quick depletion [4]. This issue is more challenging in medical applications where unreliable data collection will endanger the human life.

Due to users' mobility, it is difficult or even impossible to impose optimal central controller for the allocation of network traffic, leaving network users free to act according to their own resources. In general, the result of mutual interference amongst WBANs carries the cost of decreased network performance. Authors in [5][6] figured out the conclusion that the number of co-located WBANs, transmission power of sensors, as well as the transmission rate has a great impact on the interference level. Currently, there are mainly two types of methods to mitigate interference: interference reduction techniques and interference avoidance techniques [7]. On the one hand, Interference reduction schemes focus on minimizing the interference level at the receiver by adjusting the system parameters, such as transmission power [8][9] and modulation mode [10]. On the other hand, interference avoidance approaches aim to schedule the channel or temporal resources to available ones dynamically in WBANs [11]. However, in the scenario with multiple WBANs, the number of sensor nodes far exceeds that of the available channels, the co-channel interference is inevitable using channel scheduling [2]. Since TDMA-based scheduling methods can achieve high quality for signal transmissions, as well as low computational complexity [12][13] and WBANs put strict requirements for communication reliability, time sensitivity and energy efficiency, it is preferred to take the TDMA-based interference avoidance techniques for co-located WBANs [14][15].

The main objective of TDMA scheduling is to schedule transmissions as many as possible within every time slot under the condition that the interference among them is tolerable. The essential chanllenges are how to determine the interference among nodes and how to allocate time slots for nodes. In general, graph coloring method is a good way to establish interference sets and assign time slots for WBANs [16][17][18]. The algorithm proposed in [16] considers a coarse-grained interference model, which treats the whole WBAN as a minimum interference unit. However, sensor nodes in a WBAN may create interference with various degree, and there is no need to allocate extra resource for nodes within the interference tolerance. Hence, the work in [17] has proposed a medium-grained interference model, which considers the influence from each node in other WBANs to the whole WBAN. Furthermore, the authors of [18] have presented a fine-grained interference model, which accounts for the inter-relations from each node in other WBANs to every one in the whole WBAN. This node-level interference model can improve the spatial reuse, since it takes each node as a minimum interference set. However, all these above algorithms are implemented based on a star-topology, as well as lack of the friendly adaptation to the network QoS.

Taking the relay capability of sensor nodes and diverse QoS requirements of different 
applications into account, we propose a QoS-aware Adaptive Coloring (QAC) scheduling algorithm based on TDMA scheduling and node-level interference model, which balances the network traffic for QoS performance. Specifically, it determines interference sets by the relay-based scheme and assigns time slots by the multi-coloring approach. The main contributions of the proposed work are summarized as follows:

- Based on the node-level interference model, we present a relay scheme to determine interference sets. Then, the expected signals are strengthened due to the shortened communication distance and there are more nodes with different target receiving nodes that can transmit data in the same time slot. Thus, the sensed data can be transmitted reliably with short time delay.

- To balance the network traffic and improve resource utility, we propose a multi-coloring approach to increase the probability of obtaining more time slots for nodes with higher data rate requirements.

- In order to avoid the frequent change of time slot allocation, a launch condition for the QAC scheduling algorithm is provided, that is when the interference duration is longer than a threshold determined by the communication environment. Furthermore, a prediction model of interference duration is proposed based on the relative distance and moving speed of users.

The rest of this paper is organized as follows: Section 2 introduces the details of related works. Section 3 describes the system model and the problem formulation. The proposed QAC scheduling algorithm is depicted in Section 4. Section 5 presents the performance evaluation. Finally, this paper is concluded in Section 6.

\section{Related Work}

Our work differs from prior work primarily in its goal -- to design a QoS-aware adaptive coloring scheduling algorithm to assign time resource among multiple WBANs. To address this goal, our work liberally borrows insights from a large body of prior work in interference mitigation as discussed below.

Game theory and Optimization are the two common methods to solve the resource scheduling problem [19]. Authors in [20] proposed game theory based channel allocation approach to mitigate interference in body-to-body network scenarios. BR-SIM algorithm is developed to converge to the Nash Equilibrium solutions. A QoS-aware power control scheme based on non-cooperative game model is proposed in [21] to mitigate the interference among WBANs. Cooperative game theory model based on the Cournot competition was introduced in coexisting WBANs environment [22]. By adapting the frame structure, the interference among WBANs can be alleviated. However, the game model based methods either can't achieve the optimal solution or need to assess the opponents' strategies in advance, which are not suitable to WBANs applications.

Optimization approaches are presented to alleviate interference in WBANs [23][24]. The optimization model of [23] considers both internal interference and cross technology interference to solve the coexistence problem within the system. Authors in [24] proposed a link-quality-aware resource allocation scheme to maximize the network capacity. However, these approaches treat the reduction of interference level as the optimization goal and can't avoid the interference among WBANs.

For the optimization methods, the coloring algorithms are exploited to solve this problem. Graph coloring-based methods have been studied in many fields, such as ultra dense networks [25], vehicular ad hoc networks [26], D2D communication network [27], and wireless body 
area networks [16][17][18]. Among co-located WBANs, interference models are divided into three categories according to the size of minimum interference sets: coarse-grained, median-grained and fine-grained.

RIC proposed in [16] adopts incomplete coloring method to mitigate the inter-WBAN interference. The algorithm treats a WBAN as a minimum scheduling unit and ignores the diversity of nodes within a WBAN. The cluster-based SCA scheme [17] is based on medium-grained interference model and the node in another WBAN will be added into the interference set of the concerned WBAN if it generates interference to one of nodes in the concerned WBAN. After the interference set is formed between two WBANs, the orthogonal channels are assigned to those nodes in the interference set, and the rest of the nodes are free to use the remaining channels.

Further, a WBAN distributed coloring (WBAN-DC) algorithm based on fine-grained interference model is proposed in [18]. The algorithm is executed in two-phase. In the first phase, a global time slot is reserved in advance, in which the nodes transmit testing signals in turn. Then, the coordinator corresponding to the considered node estimates the interference signals from nodes in other WBANs one by one and if the SINR of the considered node is lower than the predefined threshold, the node creating the interference will be added into the considered node's interference set. Moreover, the nodes within a WBAN are included in the interference set of each other due to the same receiver, the coordinator. In the second phase, the coordinators implement the coloring algorithm for the interference graph. Firstly, the coordinator colors all of the nodes within its own WBAN and then communicates its coloring information with all other coordinators to decide whether its nodes can keep the proposed color based on the priority rules. The algorithm is terminated when all the nodes get colored. The algorithm is implemented based on star-topology and lack of friendly adaption to the network QoS.

The QoS-aware Adaptive Coloring (QAC) scheduling algorithm proposed in this paper consists of two sub-algorithm. In the first sub-algorithm, the coordinators determine interference sets for sensor nodes based on relay scheme. Different from the work in [18], the strength of the interference signals are estimated according to the relative distance between the transceivers, since the negative correlation relationship between them [28]. In the second sub-algorithm, the coordinators implement the proposed coloring scheme based on the interference sets, where multi-coloring will be executed following the single-coloring process to satisfy nodes' various requirements for data rate and improve the resource utility as well.

\section{System model}

In this section, we introduce the interference model used for WBANs, which is based on multi-hop topology. To avoid the frequent change of resource assignment, the launch condition for the QoS-aware Adaptive Coloring (QAC) scheduling algorithm is proposed, that is when the interference duration is longer than a threshold defined by the communication environment. Thus a prediction model for interference duration is provided as well.

\subsection{Interference model}

We consider a scenario composed of $N$ WBANs, which are located in the same geographic area of side $h$. Each WBAN is equipped with $N_{s}$ sensor nodes and a coordinator. This paper focuses on the uplink transmission scenario and nodes communicate to the corresponding coordinators directly or are relayed by other nodes within their WBANs. 


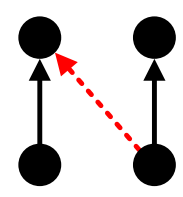

(a)

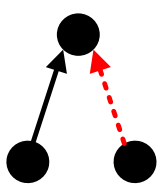

(b)

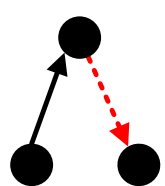

(c)

Fig. 1. Conflicting links in WBANs (the dotted lines represent the conflicting links)

The conflicting links in WBANs can be divided into three categories, as illustrated in Fig. 1. Interfering links appear between different transceiver pairs which create interference when they are scheduled concurrently, as shown in Fig. 1 (a). Intersecting links are defined as the links with the common destination, referred to Fig. 1 (b). Since the half-duplex characteristic of the sensor nodes, one node can't act as the transmitter and the receiver at the same time, as shown in Fig. 1 (c). An example is shown in Fig. 2. There are two WBANs in the area, where $\mathrm{C}_{\mathrm{i}}$ represents the coordinator of the ith WBAN, and $\mathrm{S}_{\mathrm{ij}}$ denotes the $j$ th sensor node in ith WBAN. The nodes transmit packets using constant power $\mathrm{P}_{t}$, and the circles represent the communication range of sensor nodes within which the unrelated receivers will be interfered by the transmitters and suffered from packet loss. Thus, it is obvious that link $\left(\mathrm{S}_{11}, \mathrm{C}_{1}\right)$ will interfere link $\left(\mathrm{S}_{12}, \mathrm{C}_{1}\right)$ because of the same destination. The links $\left(\mathrm{S}_{21}, \mathrm{C}_{2}\right)$ and $\left(\mathrm{S}_{22}, \mathrm{~S}_{21}\right)$ can't be activated concurrently since the sensor nodes work in half-duplex. What's more, there are interference between link $\left(\mathrm{S}_{11}, \mathrm{C}_{1}\right)$ and link $\left(\mathrm{S}_{21}, \mathrm{C}_{2}\right)$ if the SINR of node $\mathrm{S}_{21}$ is not more than the predefined threshold.

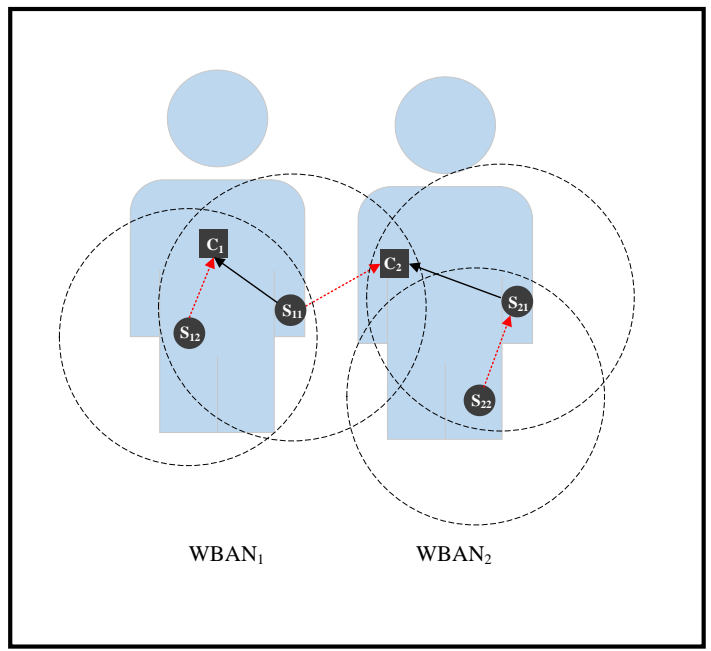

Fig. 2. Diagram of interference model for WBANs

\subsection{Interference duration prediction model}

The nature of human movements means the duration of inter-WBAN interference has a dynamic pattern. It is reasonable to assume that each WBAN user carries a smartphone due to popularity [29]. Thus, the smartphone's movement can represent the behavior of its corresponding WBAN. 


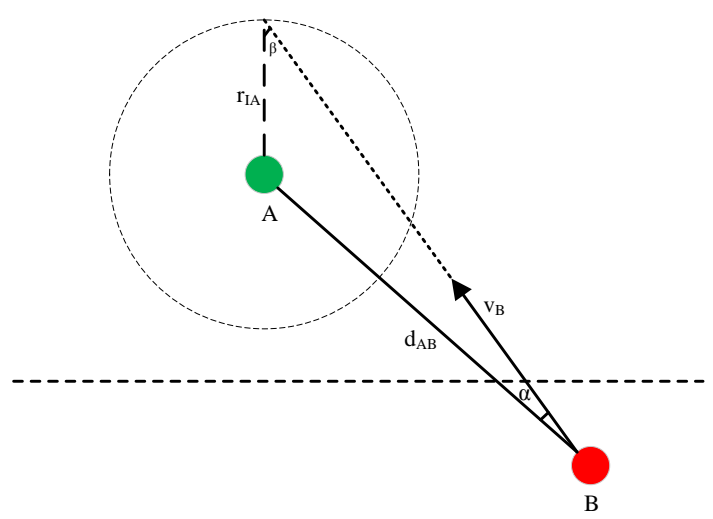

Fig. 3. Relative moving model of two WBANs

As shown in Fig. 3, there are two coexisting WBANs, A and B. The relative speed $v_{\mathrm{B}}$, deviate angel $\alpha$ and distance between them can be measured and computed using smartphones owned by the users of WBANs. The coordinator in WBAN A records the smallest signal received from its sensor nodes. Then the minimum interference-free distance $r_{\text {IA }}$ can be obtained according to formula (1).

$$
\frac{S_{\min }}{I\left(r_{I A}\right)+N_{0}}=S I N R_{t h}
$$

where $S_{\min }$ is the minimum received signal strength from sensors within WBAN A, $I\left(r_{I A}\right)$ is the maximum interference signal strength tolerated by WBAN A, which is a function of $r_{\text {IA }}$ according to the channel condition. SINR th $_{\text {in }}$ is the threshold of SINR below which the network can't work normally. $N_{0}$ is the Gaussian white noise power at the receiver. Once other WBANs step into the circle with radius $r_{\mathrm{IA}}$, they will create interference on WBAN A. Then, the interference duration $\mathrm{T}_{\text {in }}$ can be computed by formula (2).

$$
\left\{\begin{array}{l}
\frac{r_{I A}}{\sin \alpha}=\frac{d_{A B}}{\sin \beta} \\
\frac{v_{B} \cdot T_{\text {in }}}{\sin (\pi-2 \beta)}=\frac{r_{I A}}{\sin \beta}
\end{array}\right.
$$

where $d_{A B}$ and $v_{B}$ are the relative distance and moving speed between WBAN A and B, respectively. $\alpha$ and $\beta$ are geometric angles as shown in Fig. 3.

It is worth emphasizing that sensor nodes are energy limited [30]. While the coordinators are energy sufficient because they usually are smartphones or PADs, etc. In order to prolong the network lifetime, the coordinators do all the work during the execution of the scheduling algorithm. 


\section{QoS-aware Adaptive Coloring (QAC) scheduling algorithm}

In this section, we first introduce the execution procedure of the proposed QAC scheduling algorithm. Then, an example is given to illustrate how the algorithm works. Finally, we present the launch condition of the algorithm to avoid frequent resource scheduling among coexisting WBANs.

\subsection{Algorithm description}

With the goal of rescheduling time slots among co-located WBANs, the proposed QAC scheduling algorithm includes two sub-algorithm -- interference sets determination and time slots assignment.

The pseudo-code of the first sub-algorithm is shown in Algorithm 1. The channel characteristics of on-body and off-body are different [31], the algorithm considers the internal and external nodes of the interested WBAN, respectively, as shown in Step 1 and Step 2 of Algorithm 1. Since there is a negative correlation between the strength of the received signal and the distance between transceivers, the interference power between sensors can be estimated by the distance between them. Specifically, in each step, the nodes are sorted by descending order of the relative distance to the target receiving node of the considered node firstly. Then the first node in the sequence sends a testing signal and the target receiving node measures the interference power. Thus, the SINR of the considered node can be computed and whether the first node of the sequence belongs to the interference set of the considered node can be determined.

The basic idea is that if SINR of the considered node is not more than the predefined threshold under the interference of the first node in the sequence, the first node will be added into the interference set of the considered node and other nodes in the sequence closer to the target receiving node than the first node will be included in the interference set as well. Otherwise, the first node is deleted and the sequence is updated.

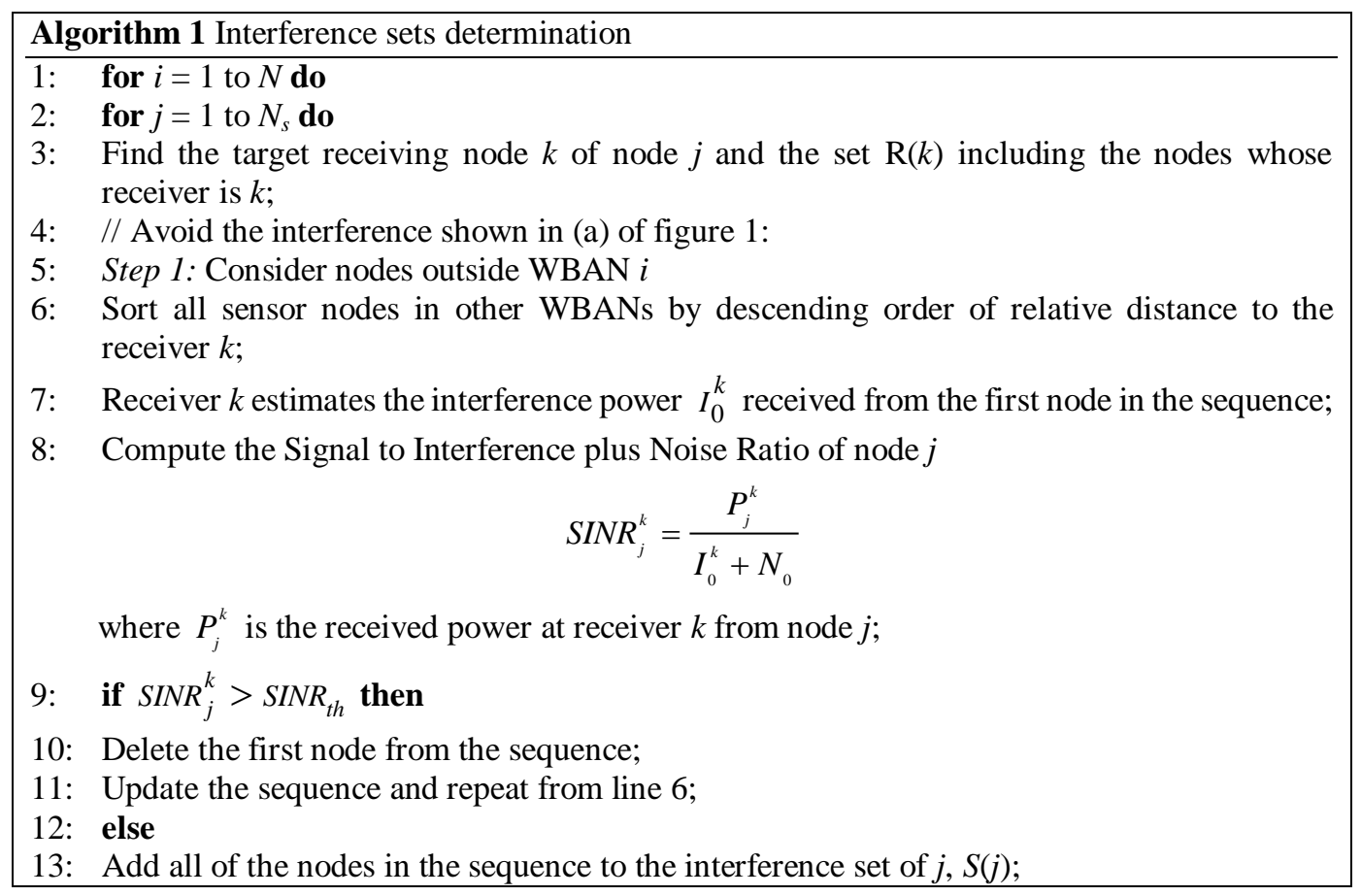




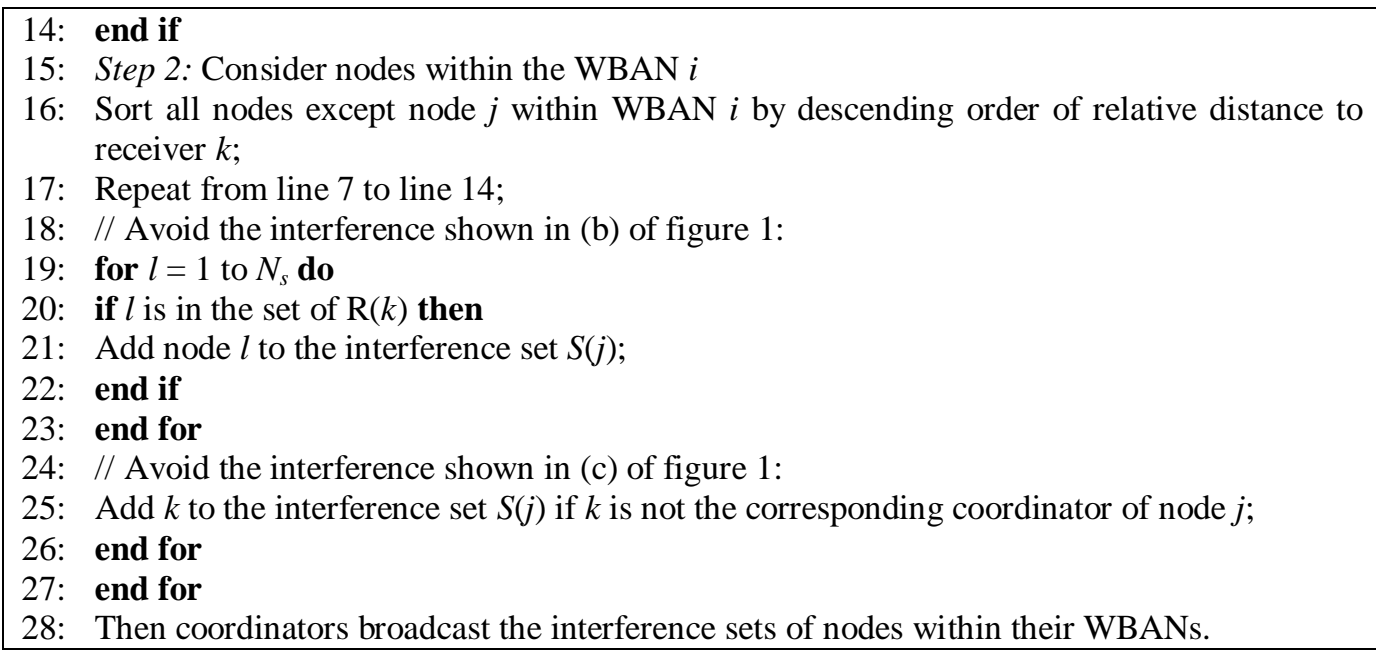

In the proposed QAC algorithm, the target receiving node of the considered node will be activated only when it needs to receive signals from the considered node or from the first node in the sequence, whereas the target receiving node with WBAN-DC [18] always keeps active during the period of interference sets determination for measuring interference signal from each other node. Thus, the proposed algorithm is more energy efficient since the reduction of active time can significantly reduce the power consumption of a sensor node.

By connecting the nodes with those in their interference sets, the interference graph is constructed. Then, the coordinators update time slots assignment for nodes by implementing the coloring scheme as shown in Algorithm 2.

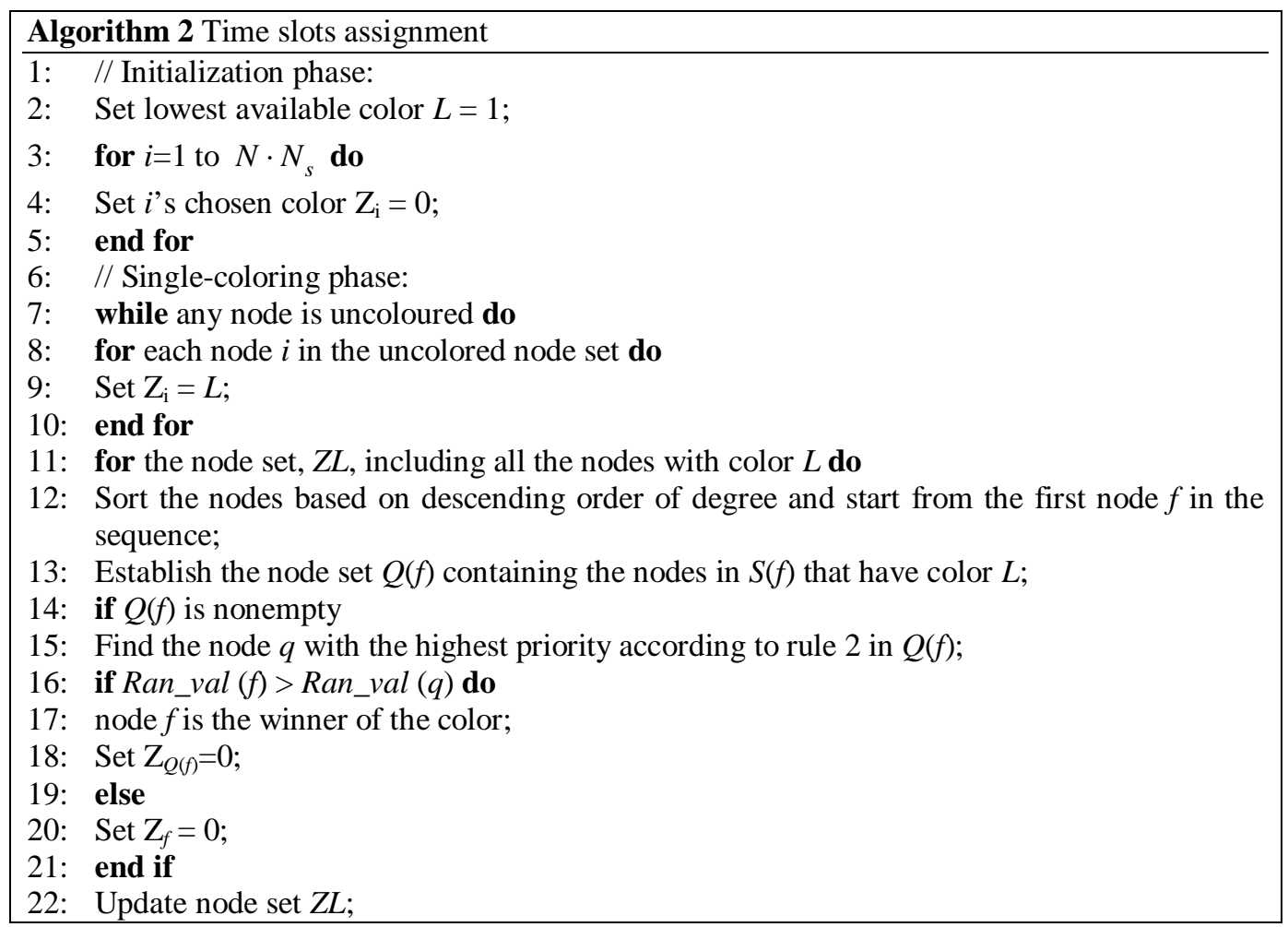




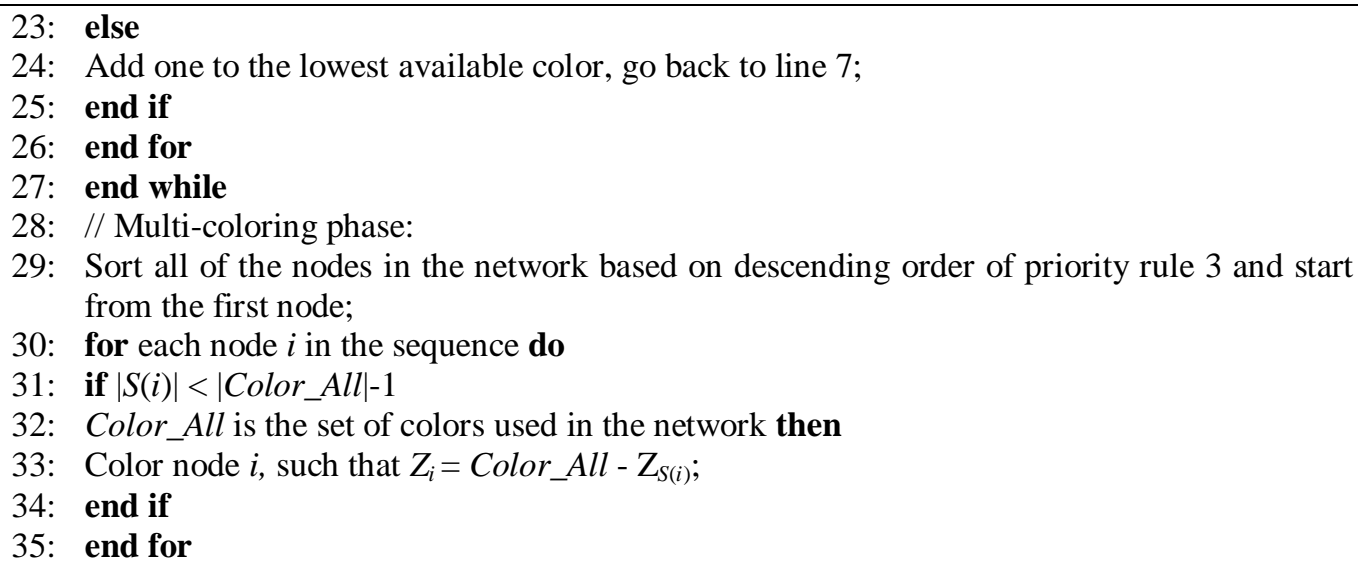

Three kinds of priority rules are exploited to solve the conflicts of color assignment among neighboring nodes:

(1) Node's degree, which can be calculated as follows for a given node $v_{i}$ :

$$
\operatorname{deg}\left(v_{i}\right)=\sum_{j=1}^{m \cdot N_{s}} a\left(v_{i}, v_{j}\right) \cdot b\left(v_{i}, v_{j}\right)
$$

where $a\left(v_{i}, v_{j}\right)=\left\{\begin{array}{ll}1, & \left(v_{i}, v_{j}\right) \in E \\ 0, & \text { otherwise }\end{array}, b\left(v_{i}, v_{j}\right)=\left\{\begin{array}{ll}1, & C_{v_{i}} \cap C_{v_{j}} \neq \varnothing \\ 0, & \text { otherwise }\end{array}\right.\right.$.

(2) Random number assigned to a node, which is unique for each node in the network.

(3) Date rate requirement of a node, which is different among nodes with different functions, as shown in Table 1.

Specifically, the algorithm considers that the priority of node $v_{1}$ is higher than $v_{2}$ if any of the following rules is satisfied:

- Rule 1: $\operatorname{deg}\left(v_{1}\right)>\operatorname{deg}\left(v_{2}\right)$

- Rule 2: Ran_val $\left(v_{1}\right)>$ Ran_val $\left(v_{2}\right)$

- Rule 3: rate $\left(v_{1}\right)>$ rate $\left(v_{2}\right)$

where $\operatorname{deg}(v)$ is the degree of node $v$, Ran_val $(v)$ is the random value assigned to $v$ and rate $(v)$ represents the data rate requirement of physiological information collected by node $v$.

Table 1. Data rate of different type of sensors [32]

\begin{tabular}{|c|c|}
\hline Sensor type & Data rate(bps) \\
\hline \hline ECG & $71-288 \times 10^{3}$ \\
\hline Pulse oximeter & 16 \\
\hline Gyroscope insulin actuator & 1600 \\
\hline Temperature sensor & 120 \\
\hline Accelerometer & 35 \\
\hline
\end{tabular}

The highlight of the coloring algorithm is that coloring all nodes with colors as few as possible. In the algorithm proposed in this paper, the nodes in all WBANs form an integral network and the coordinators can color their nodes without any conflict by negotiating with each other in advance, instead of first coloring the nodes within their WBANs and then 
deciding whether the nodes can keep the proposed color by exchanging information with other coordinators proposed in [18]. The coloring procedure is depicted in Algorithm 2:

In the process of initialization, the lowest available color which indicated by digits starting from one is set for nodes. Then, all the uncolored nodes in the network obtain the lowest available color and the node with the highest priority according to rule 1 is found as shown in line 12 of Algorithm 2. If the color of the node doesn't clash with that of its neighbors, or the node has the highest priority level based on the priority rule 2 amongst its neighbors, it will win the color. Otherwise, the node loses the color and waits for being colored in the subsequent round, as indicated in the single-coloring phase of Algorithm 2. The procedure will not be terminated until all of the nodes in the network are colored without collision. In order to ensure the network QoS, the nodes with higher requirements of data rate have higher probability of getting multiple colors. At last, the coordinators assign corresponding time slots to their nodes.

\subsection{A walk-through example}

We use an example to illustrate how the QAC scheduling algorithm works. As shown in Fig. 4, there are two WBANs, where each WBAN consists of one coordinator and three sensor nodes. It can be seen that node $\mathrm{A}$ in $\mathrm{WBAN}_{1}$ communicates to the coordinator by two hops.

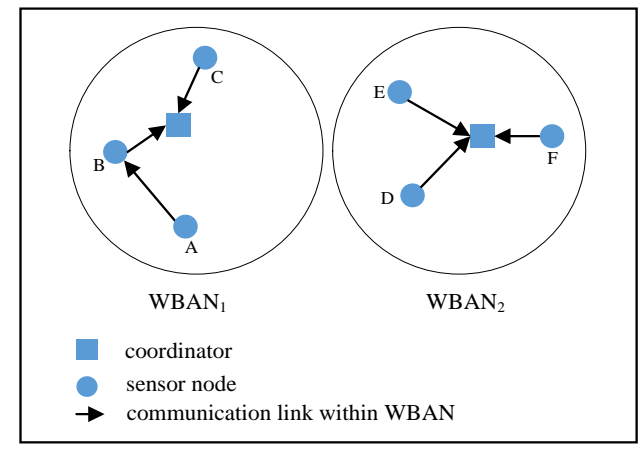

Fig. 4. A demonstration of the communication model of WBANs

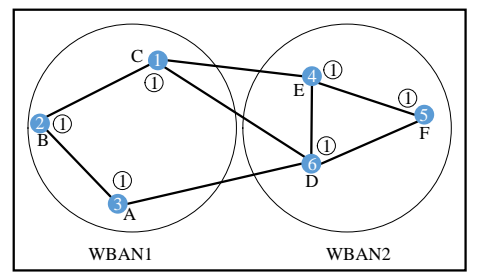

(a)

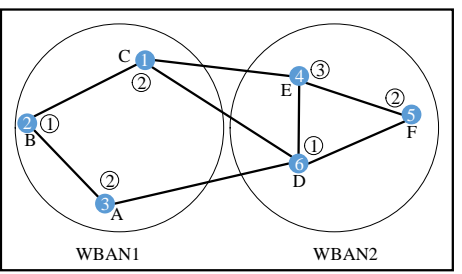

(c)

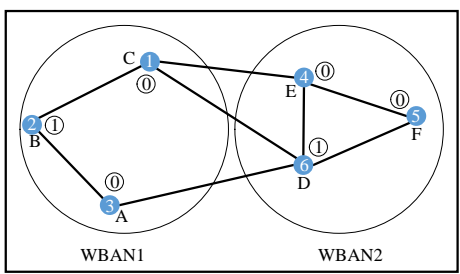

(b)

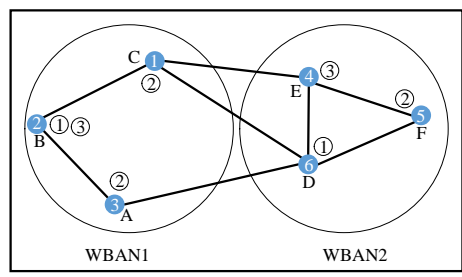

(d)

Fig. 5. A demonstration of the QAC scheduling algorithm 
As demonstrated in Fig. 5, by estimating the strength of the interference signal, each coordinator can determine the interference sets for its nodes and construct the interference graph showed in Fig. 5 (a). The numbers labeled on the nodes represent the random values assigned to nodes. Firstly, all uncolored nodes are colored with the lowest available color $L=1$. It can be seen that node $\mathrm{D}$ has the highest priority among all nodes according to rule 1 . Then, start from node $\mathrm{D}$ and find its neighbor nodes' set $Q(D)=\{A, C, E, F\}$. Compare the priority of node $\mathrm{D}$ with its neighbor nodes based on rule 2, it can be found that node $\mathrm{D}$ will win the color because of its highest priority and its neighbor nodes loss the color at the same time. Refer to Fig. 5 (b), after the first round of the proposed algorithm, nodes B and D remain the color and there is no conflict in the network. Then, add one to the lowest available color, i.e. $L=2$. The above procedure will be repeated until all of the nodes are colored, as shown in Fig. 5 (c). At last, the multi-coloring phase is executed. we assume node $B$ has the highest priority among all of the nodes according to rule 3. It can be seen that node B can be colored by two colors and there is no more available color for other nodes in the network. The result after completing the coloring algorithm is showed in Fig. $\mathbf{5}$ (d). Each color corresponds to a special time slot and nodes with the same color will be activated simultaneously.

\subsection{Launch condition of QAC scheduling algorithm}

Interference has a negtive effect on communication quality, which can be evaluated by the packet loss. The execution of scheduling algorithm will also lead to extra packet loss. Therefore, the launch condition of the QAC scheduling algorithm is that the packet loss caused by the interference is more than that resulted from the implementation of the scheduling algorithm, which can be expressed by formula (4) mathematically. The notations used are defined in Table 2.

$$
T_{0} \cdot P R+P L R_{A} \cdot\left(T_{\text {in }}-T_{0}\right)<T_{\text {in }} \cdot P L R_{I}
$$

Table 2. List of notations

\begin{tabular}{|c|c|}
\hline Notation & Definition \\
\hline \hline$T_{0}$ & Required time for performing Algorithm 1 and Algorithm 2 \\
\hline$T_{i n}$ & Interference duration \\
\hline$P R$ & Transmission rate of data packets \\
\hline$P L R_{I}$ & Packet loss rate under interference \\
\hline$P L R_{A}$ & Packet loss rate in interference free condition \\
\hline
\end{tabular}

It is expected that the packet transmission rate will be zero when performing the Algorithm 1 and Algorithm 2, and the $P L R_{I}$ can be estimated by the SINR of the considered node [33]. The packet loss rate in interference free condition is mainly caused by multipath, instability of links, etc., which can be neglected compared with $P L R_{I}$ or $P R$. Therefore, the formula (5) can be derived.

$$
T_{i n}>\frac{T_{0} \cdot\left(P R-P L R_{A}\right)}{P L R_{I}-P L R_{A}} \approx \frac{T_{0} \cdot P R}{P L R_{I}}=T_{t h}
$$

It can be observed that the threshold $T_{t h}$ can be defined as a function of $T_{0}, P R, P L R_{I}$, which describe the communication environment in this paper. Thus, the QAC scheduling algorithm will be launched when the interference duration is greater than the threshold. 
For a network with $\mathrm{N}$ co-located WBANs and $\mathrm{N}_{\mathrm{s}}$ nodes in each WBAN, the time complexity of the proposed QAC scheduling algorithm is $\mathrm{O}\left(N \cdot N_{s}\right)$. Thus, it can be inferred that the execution of the algorithm will result in a very small amount of packet loss. Then if the packet loss caused by interference is less than that brought by the implementation of the algorithm (i.e., the interference duration $T_{i n}$ is shorter than the threshold $T_{t h}$ ), the interference can be neglected since it has little effect on the communication quality.

\section{Performance evaluations}

\subsection{Simulation setup}

In this section, Monte Carlo simulation is used to evaluate the performance of the proposed QAC scheduling algorithm. The data points in the figures are the average value of simulation under 5000 random topologies. In the simulation, there are ten sensor nodes in each WBAN, i.e. $\mathrm{N}_{\mathrm{s}}=10$. We assume that the WBANs are located in a square of side $h$ randomly. For simplicity, we use the two-hop topology as an example to evaluate the performance of the QAC scheduling algorithm. The source node chooses the node which is closer to the coordinator as its relay node to prolong its lifetime. It should be emphasized that the proposed algorithm can be extended to arbitrary topology based on the same principle.

To evaluate the efficiency of the QAC scheduling algorithm, we propose the relay-based single coloring (RSC) algorithm and single-hop single coloring (SHSC) algorithm. The only difference between RSC algorithm and QAC scheduling algorithm is that the former has no multi-coloring phase. The SHSC algorithm is implemented based on star-topology and there is no multi-coloring phase either. The coloring method of SHSC is the same with single-coloring phase of QAC scheduling algorithm.

In the simulation, we compare the QAC scheduling algorithm with RSC algorithm, SHSC algorithm and WBAN-DC algorithm proposed in [18] which is the most similar algorithm to the QAC scheduling algorithm to the best of our knowledge.

\subsection{Simulation results}

We focus mainly on the performance of network capacity, average delay and resource utility. (1) Network Capacity is formulated by formula (6), where the SINR $_{i}$ indicates the SINR of node $i$. (2) Average Delay is defined as the minimum number of time slots used for all nodes in the network to active once [34], which is a critical metric for WBANs with higher requirement for real-time performance of data transmission. (3) The resource utility is well characterized by the average number of vertexes painted by each color (VPC) [16].

$$
C_{p}=\sum_{i=1}^{m \cdot N_{s}} \log _{2}\left(1+S I N R_{i}\right)
$$

\subsubsection{Evaluation of network capacity}

First, we examine the network capacity with the number of co-located WBANs varies from 2 to 10 . 


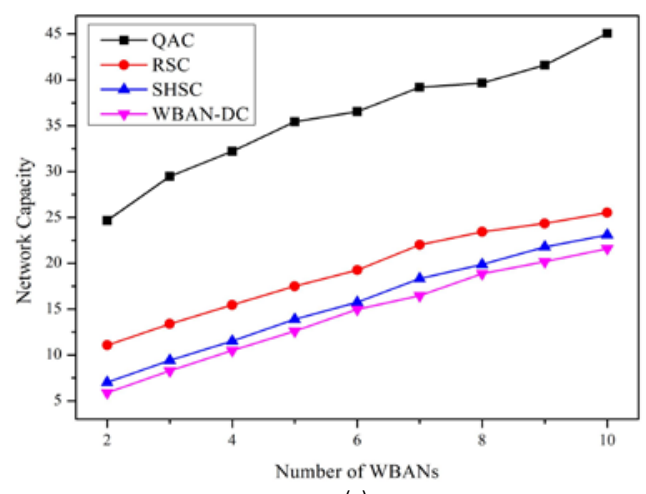

(a)

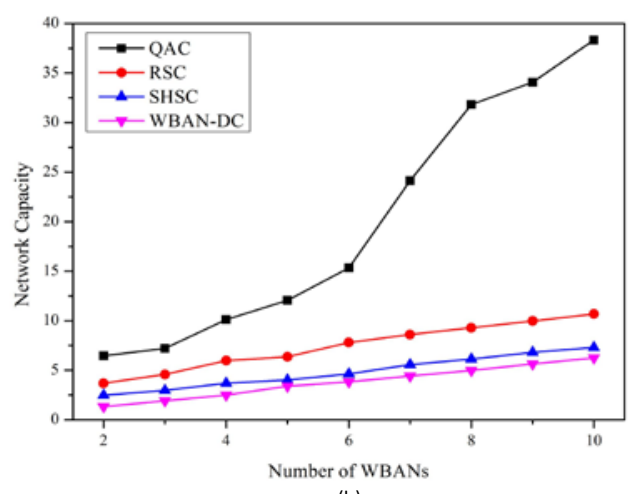

(b)

Fig. 6. Network capacity versus the number of WBANs coexisting in a square area of side $h=6$.

(a) The transmission power is set to $0 \mathrm{dBm}$

(b) The transmission power is set to $-20 \mathrm{dBm}$

As shown in Fig. 6, the points showed in Fig. 6 (a) and Fig. 6 (b) are obtained when the transmission power of sensor nodes are $0 \mathrm{dBm}$ and $-20 \mathrm{dBm}$, respectively. It can be observed that the QAC scheduling algorithm proposed in this paper outperforms the other algorithms. The performance of QAC is better than RSC, which implies that the proposed multi-coloring method could improve the network capacity substantially by allowing nodes with higher requirements of data rate to be active in more than one time slot. The network capacity of using RSC is better than that using SHSC. The reason is that the relay-based scheme is used in RSC, such that there are more nodes have distinct target receivers that are allowed to transmit data at the same time slot and the expected signals are strengthened due to the shortened communication distance. It can be inferred that the coloring method proposed in this paper is more efficient in improving the network capacity by comparing SHSC with WBAN-DC. In a word, the proposed QAC scheduling algorithm based on relay scheme and multi-coloring approach can improve the network capacity dramatically. Thus, the reliability and real-time of data transmission are improved. Furthermore, with the growth of the number of WBANs, the network capacity increases since more active nodes coexist in the area.

Through comparing the simulation results in Fig. 6 (a) and Fig. 6 (b), it can be figured out the condition that it is a good way to improve the network capacity by increasing the transmission power. The reason is that as the transmission power increases, the strength of expected signal is enhanced and then the SINR of the node is increased.

\subsubsection{Evaluation of average delay}

As demonstrated in Fig. 7 (a), QAC and RSC have the same average delay. The reason is that the same coloring method used in single-coloring phase results in the same number of colors used. It is expected that SHSC is better than WBAN-DC due to the better coloring approach proposed in SHSC, as shown in Fig. 7 (b). Since more nodes can be tolerated in one time slot with the proposed QAC scheduling algorithm, the average delay by using QAC is optimized by almost 4 times of that using WBAN-DC. Thus, the sensed data can be transmitted timely with the proposed algorithm.

The received signal is strengthened with the increase of transmission power. Then more nodes can be accommodated to transmit concurrently. Thus, compared with the network in which the nodes' transmission power is set to $-20 \mathrm{dBm}$, the network with higher transmission power, i.e., $0 \mathrm{dBm}$, can achieve smaller delay, as illustrated in Fig. 7 (a) and Fig. 7 (b). 
Moreover, the average delay increases with the rise of the number of WBANs because of more serious interference caused by denser nodes.
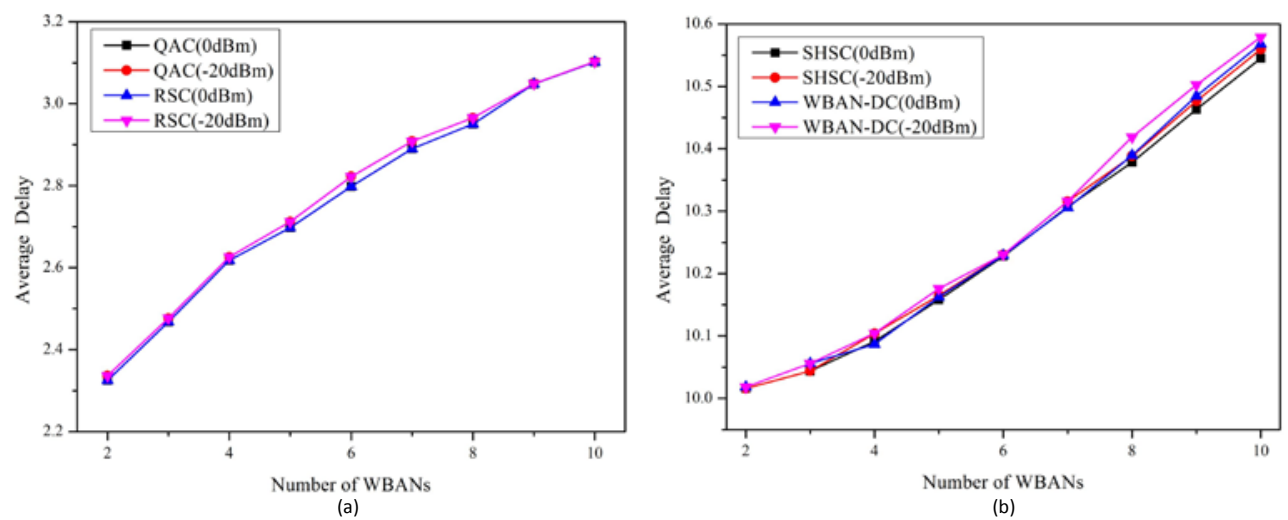

Fig. 7. Average delay versus the number of WBANs coexisting in a square area of side $h=6$.

(a) The transmission power is set to $0 \mathrm{dBm}$

(b) The transmission power is set to $-20 \mathrm{dBm}$

\subsubsection{Evaluation of resource utility}

Fig. 8 compares the VPC of QAC with the other algorithms. Though QAC and RSC occupy the same number of time slots for all nodes in the network activated once, the number of nodes that are active in one time slot by implementing QAC is approximately twice of that with RSC when the number of co-located WBANs is more than three. The reason is that the multi-coloring is used in QAC, then the nodes with higher data rate requirements can obtain more than one time slot. It is demonstrated that the VPC using SHSC and WBAN-DC is quite low, which is coincidence with the above analysis that they need more colors compared with other algorithms. The network performance of Fig. 8 (a) is better than Fig. 8 (b) because of the anti-interference capability is improved by enhancing the transmission power.
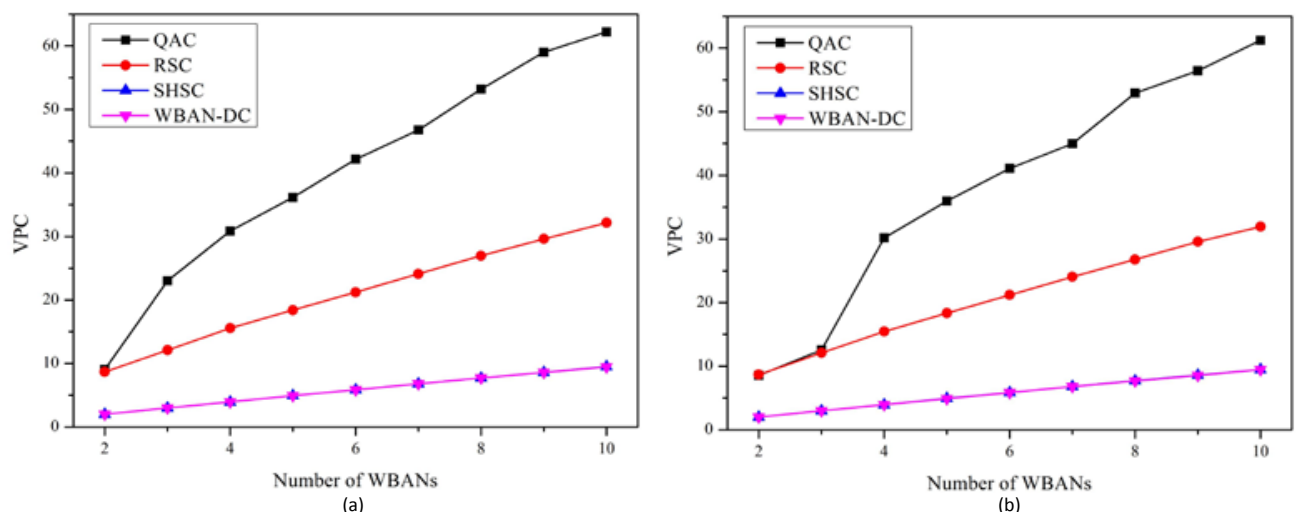

Fig. 8. VPC versus the number of WBANs coexisting in a square area of side $h=6$.

(a) The transmission power is set to $0 \mathrm{dBm}$

(b) The transmission power is set to $-20 \mathrm{dBm}$

Fig. 9 shows the effect of the size of the area accommodating WBANs on the VPC. There are 5 WBANs located in the area and the transmission power of nodes is set to $0 \mathrm{dBm}$ in the scenario. The figure shows that increasing $h$ improves the VPC. For fixed number of WBANs 
placed in the area randomly, increasing the side length will enlarge the average distance between WBANs and weaken the interference signals. Then more nodes could be activated in the same time slot, and the VPC is improved. As it can be seen, when the area is large enough such that the interference level is quite low, the network performance will not be improved too much with the area expansion.

It can be observed that the VPC of QAC is twice of RSC and nearly eight times of both SHSC and WBAN-DC, since the implementation of the proposed relay-based scheme and multi-coloring approach can improve the resource utility effectively.

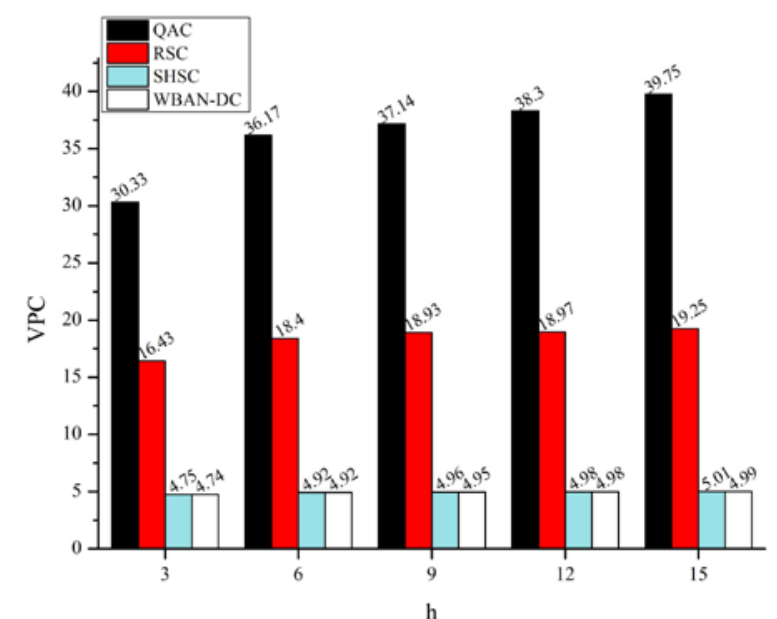

Fig. 9. VPC versus the square side $h$ when the transmission power is set to $0 \mathrm{dBm}$.

Based on the above analysis, it can be concluded that the propsoed QAC scheduling algorithm is capable of improving the reliability and real-time of data transmission, which is of significance for WBANs medical applications monitoring life-critical physiological parameters. Moreover, the diverse data rate requirements of sensed data are satisfied by the multi-coloring approach. Further, the proposed algorithm enhances the resource utility greatly, which enables more co-located WBANs to work normally in densely populated areas, such as the waiting rooms in a hospital or subway stations. In addition, a sensor can increase its transmission power when abnormal data is collected from the patient to further improve the reliability and real-time of critical data transmission.

\section{Conclusion}

In this work, a novel QoS-aware Adaptive Coloring (QAC) scheduling algorithm is proposed for resource scheduling among coexisting WBANs. There is no limitation on topology for the algorithm implementation. The QAC scheduling algorithm is capable of improving the reliability and real-time of data transmission by using the relay-based scheme and the multi-coloring approach. Moreover, the diverse data rate requirements of nodes are satisfied by assigning different number of time slots to the nodes according to their demands, which is critical for applications with stringent QoS requirements, such as health monitoring. To maintain systematic stability and increase the reliability of communication, the algorithm is executed only when the interference duration between WBANs is greater than a threshold determined by the communication environment. The simulation study shows that more WBANs are clustered together, more serious interference will be generated. The QAC 
scheduling algorithm can make WBANs work normally in densely populated areas by improving the resource utility, which is of significance for the application and popularization of WBANs.

This work only focuses on the scenario that the WBANs are placed randomly. In the future, we would like to analyze more practical scenarios, such as in the coffee house, on the street or in the railway station, which have different topologies. The effect of human activity on the network performance will be further considered as well as the implementation of QAC scheduling algorithm in the real world.

\section{Acknowledgement}

This research was supported by National Natural Science Foundation of China (No. 61372118).

\section{References}

[1] R. Cavallari, F. Martelli, R. Rosini, C. Buratti and R. Verdone, "A survey on wireless body area networks: technologies and design challenges," IEEE Communications Surveys \& Tutorials, vol. 16, no. 3, pp. 1635-1657, 2014. Article (CrossRef Link).

[2] S. Movassaghi, M. Abolhasan, J. Lipman, D. Smith and A. Jamalipour, "Wireless body area networks: A survey,” IEEE Communications Surveys \& Tutorials, vol. 16, no. 3, pp. 1658-1686, 2014. Article (CrossRef Link).

[3] M. Salayma, A. Al-Dubai, I. Romdhani and Y. Nasser, "Wireless Body Area Network (WBAN): A Survey on Reliability, Fault Tolerance, and Technologies Coexistence," ACM Computing Surveys (CSUR), vol. 50, no. 1, pp. 3, 2017. Article (CrossRef Link).

[4] S. Huang, J. Cai, H. Chen and F. Zhao, "Low-complexity priority-aware interference-avoidance scheduling for multi-user coexisting wireless networks," IEEE Transactions on Wireless Communications, vol. 17, no. 1, pp. 112-126, 2018. Article (CrossRef Link).

[5] B. de Silva, A. Natarajan and M. Motani, "Inter-user interference in body sensor networks: Preliminary investigation and an infrastructure-based solution," in Proc. of Sixth International Workshop on Wearable and Implantable Body Sensor Networks (BSN), Berkeley, USA, pp. 35-40, 2009. Article (CrossRef Link).

[6] P. H. Ghare and A. G. Kothari, "Interference Analysis and Mitigation Techniques in Wireless Body Area Networks,” Wireless Personal Communications, pp. 1-12, 2017. Article (CrossRef Link).

[7] S. Movassaghi, A. Majidi, A. Jamalipour, D. Smith and M. Abolhasan, "Enabling interference-aware and energy-efficient coexistence of multiple wireless body area networks with unknown dynamics,” IEEE Access, vol. 4, pp. 2935-2951, 2016. Article (CrossRef Link).

[8] R. Kazemi, R. Vesilo, E. Dutkiewicz and G. Fang, "Inter-network interference mitigation in wireless body area networks using power control games," in Proc. of 2010 International Symposium on Communications and Information Technologies, Tokyo, Japan, pp. 81-86, 2010. Article (CrossRef Link).

[9] L. Zou, B. Liu, C. Chen and C. W. Chen, "Bayesian game based power control scheme for inter-WBAN interference mitigation," in Proc. of Global Communications Conference (Globecom), Austin, USA, pp. 240-245, 2014. Article (CrossRef Link).

[10] R. Kumar and S. Das, "Interference mitigation in wireless body area networks using modified and modulated MHP,” Wireless personal communications, vol. 77, no. 2, pp. 1343-1361, 2014. Article (CrossRef Link).

[11] N. Thepvilojanapong, S. Motegi, A. Idoue and H. Horiuchi, "Adaptive channel and time allocation for body area networks,” IET Communications, vol. 5, no. 12, pp. 1637-1649, 2011.

Article (CrossRef Link). 
[12] S. Movassaghi, M. Abolhasan, D. Smith and A. Jamalipour, "AIM: Adaptive Internetwork interference mitigation amongst co-existing wireless body area networks," in Proc. of Global Communications Conference (Globecom), Austin, USA, pp. 2460-2465, 2014.

Article (CrossRef Link).

[13] H. ElSawy, E. Hossain and S. Camorlinga, "Spectrum-Efficient Multi-Channel Design for Coexisting IEEE 802.15. 4 Networks: A Stochastic Geometry Approach,” IEEE Transactions on Mobile Computing, vol. 13, no. 7, pp. 1611-1624, 2014. Article (CrossRef Link).

[14] S. Ergen and P. Varaiya, "PEDAMACS: Power efficient and delay aware medium access protocol for sensor networks,” IEEE Transactions on Mobile Computing, vol. 5, no. 7, pp. 920-930, 2006. Article (CrossRef Link).

[15] M. Salayma, A. Al-Dubai, I. Romdhani and Y. Nasser, "New dynamic, reliable and energy efficient scheduling for wireless body area networks (WBAN)," in Proc. of International Conference on Communications (ICC), Washington, USA, pp. 1-6, 2017. Article (CrossRef Link).

[16] S. Cheng and C. Huang, "Coloring-based inter-WBAN scheduling for mobile wireless body area networks,” IEEE Transactions on Parallel and Distributed Systems, vol. 24, no. 2, pp. 250-259, 2013. Article (CrossRef Link).

[17] S. Movassaghi, M. Abolhasan and D. Smith, "Cooperative scheduling with graph coloring for interference mitigation in wireless body area networks," in Proc. of Wireless Communications and Networking Conference (WCNC), Istanbul, Turkey, pp. 1691-1696, 2014. Article (CrossRef Link).

[18] W. Huang and T. Quek, "On constructing interference free schedule for coexisting wireless body area networks using distributed coloring algorithm," in Proc. of 12th International Conference on Wearable and Implantable Body Sensor Networks (BSN), Cambridge, USA, pp. 1-6, 2015. Article (CrossRef Link).

[19] T. Le and S. Moh, "Interference Mitigation Schemes for Wireless Body Area Sensor Networks: A Comparative Survey,” Sensors, vol. 15, no. 6, pp. 13805-13838, 2015. Article (CrossRef Link).

[20] A. Meharouech, J. Elias and A. Mehaoua, "A two-stage game theoretical approach for interference mitigation in Body-to-Body Networks,” Computer Networks, vol. 95, pp. 15-34, 2016. Article (CrossRef Link).

[21] Y. Li, J. Pan and X. Tian, “A Utility-Based and QoS-Aware Power Control Scheme for Wireless Body Area Networks,” KSII Transactions on Internet and Information Systems, vol. 10, no. 9, 2016. Article (CrossRef Link).

[22] S. Shin, W. Su and J. Cho, "A game theory model to support QoS in overlapped WBAN environment," in Proc. of the 6th International Conference on Ubiquitous Information Management and Communication (ICUIMC), Kuala Lumpur, Malaysia, pp. 47, 2012. Article (CrossRef Link).

[23] J. Elias, S. Paris and M. Krunz, "Cross-Technology Interference Mitigation in Body Area Networks: An Optimization Approach,” IEEE Transactions on Vehicular Technology, vol. 64, no. 9, pp. 4144-4157, 2015. Article (CrossRef Link).

[24] A. Samanta, S. Bera and S. Misra, "Link-Quality-Aware Resource Allocation with Load Balance in Wireless Body Area Networks,” IEEE Systems Journal, vol. 99, pp. 1-8, 2015. Article (CrossRef Link).

[25] C. Zhao, X. Xu, Z. Gao and L. Huang, “A coloring-based cluster resource allocation for ultra dense network," in Proc. of International Conference on Signal Processing, Communications and Computing (ICSPCC), Hong Kong, China, pp. 1-5, 2016. Article (CrossRef Link).

[26] T. Yang, R. Zhang, X. Cheng and L. Yang, "A graph coloring resource sharing scheme for full-duplex cellular-VANET heterogeneous networks," in Proc. of International Conference on Computing, Networking and Communications (ICNC), Hawaii, USA, pp. 1-5, 2016. Article (CrossRef Link).

[27] T. Yang, R. Zhang, X. Cheng and L. Yang, "Graph Coloring Based Resource Sharing (GCRS) Scheme for D2D Communications Underlaying Full-Duplex Cellular Networks,” IEEE Transactions on Vehicular Technology, vol. PP, no. 99, pp. 1, 2017. Article (CrossRef Link). 
[28] X. Wang and L. Cai, “Interference analysis of co-existing wireless body area networks,” in Proc. of Global Communications Conference (Globecom), Houston, USA, pp. 1-5, 2011. Article (CrossRef Link).

[29] A. S. Abiodun, M. H. Anisi, I. Ali, A. Akhunzada and M. K. Khan, "Reducing power consumption in wireless body area networks: a novel data segregation and classification technique,” IEEE Consumer Electronics Magazine, vol. 6, no. 4, pp. 38-47, 2017. Article (CrossRef Link).

[30] G. Abdul-Salaam, A. H. Abdullah and M. H. Anisi, "Energy-efficient data reporting for navigation in position-free hybrid wireless sensor networks," IEEE Sensors Journal, vol. 17, no. 7, pp. 2289-2297, 2017. Article (CrossRef Link).

[31] D. Smith, L. Hanlen, J. Zhang, D. Miniutti, D. Rodda and B. Gilbert, "First-and second-order statistical characterizations of the dynamic body area propagation channel of various bandwidths," annals of telecommunications-annales des telecommunications, vol. 66, no. 3-4, pp. 187-203, 2011. Article (CrossRef Link).

[32] S. Misra and S. Sarkar, "Priority-based time-slot allocation in wireless body area networks during medical emergency situations: An evolutionary game-theoretic perspective,” IEEE Journal of Biomedical and Health Informatics, vol. 19, no. 2, pp. 541-548, 2015. Article (CrossRef Link).

[33] IEEE Std 802.15.4, "IEEE Standard for Information technology -- Local and metropolitan area networks -- Specific requirements -- Part 15.4: Wireless Medium Access Control (MAC) and Physical Layer (PHY) Specifications for Low Rate Wireless Personal Area Networks (WPANs),” pp. 1-320, 2006. Article (CrossRef Link).

[34] M. Sharif and B. Hassibi, "Delay considerations for opportunistic scheduling in broadcast fading channels," IEEE Transactions on Wireless Communications, vol. 6, no. 9, pp. 3353-3363, 2007. Article (CrossRef Link). 


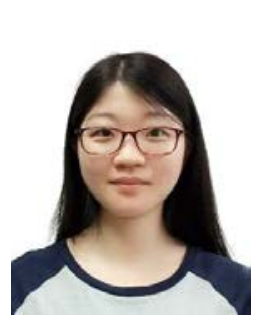

Jingxian Wang received the B.S. degree in Information and Communication Engineering from Harbin Engineering University, Heilongjiang. She is currently pursuing the Ph.D. degree in the School of Information and Communication Engineering, Beijing University of Posts and Telecommunications (BUPT), China. Her main research interest is wireless body area networks.

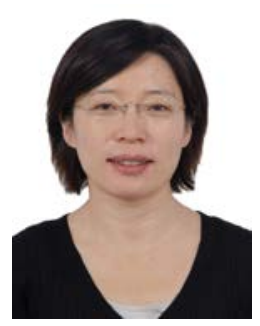

Yongmei Sun received her Ph.D. degree in Information and Communication Engineering from the University of Tokyo, Japan, in 2006. Currently she is a professor at Beijing University of Posts and Telecommunications (BUPT), China. Her research interests include optical networks and sensor networks.

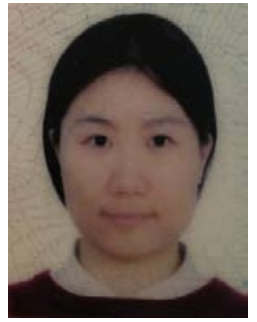

Shuyun Luo received her Ph.D. degree in communication and information system from Beijing University of Posts and Telecommunications (BUPT), China, in 2016. She is currently an assistant professor in the College of Computer Science and Technology, Zhejiang Sci-tec University, China. Her research interests include edge computing, ad hoc and sensor networks and network economics.

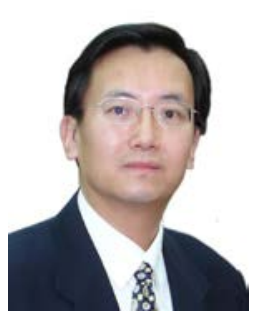

Yuefeng Ji is a professor at the School of Information and Communication Engineering, Beijing University of Posts and Telecommunications, Beijing, China. His research interests are primarily in the areas of broadband telecommunication system and networks, with emphasis on the key theory, realization of technology, and applications. 